ARTICLE

\title{
Chirality transmission in macromolecular domains
}

\author{
Shankar Pandey 1,7, Shankar Mandal1,7, Mathias Bogetoft Danielsen (1) 2,7, Asha Brown ${ }^{3}$, Changpeng Hu', \\ Niels Johan Christensen ${ }^{4}$, Alina Vitaliyivna Kulakova ${ }^{5}$, Shixi Song ${ }^{2}$, Tom Brown (D) ${ }^{6}$, Knud J. Jensen (i) ${ }^{4}$, \\ Jesper Wengel ${ }^{2}$, Chenguang Lou (10) ${ }^{2 \otimes} \&$ Hanbin Mao (i) ${ }^{1 凶}$
}

Chiral communications exist in secondary structures of foldamers and copolymers via a network of noncovalent interactions within effective intermolecular force (IMF) range. It is not known whether long-range chiral communication exists between macromolecular tertiary structures such as peptide coiled-coils beyond the IMF distance. Harnessing the high sensitivity of single-molecule force spectroscopy, we investigate the chiral interaction between covalently linked DNA duplexes and peptide coiled-coils by evaluating the binding of a diastereomeric pair of three DNA-peptide conjugates. We find that right-handed DNA triple helices well accommodate peptide triple coiled-coils of the same handedness, but not with the left-handed coiled-coil stereoisomers. This chiral communication is effective in a range $(<4.5 \mathrm{~nm})$ far beyond canonical IMF distance. Small-angle X-ray scattering and molecular dynamics simulation indicate that the interdomain linkers are tightly packed via hydrophobic interactions, which likely sustains the chirality transmission between DNA and peptide domains. Our findings establish that long-range chiral transmission occurs in tertiary macromolecular domains, explaining the presence of homochiral pairing of superhelices in proteins.

\footnotetext{
${ }^{1}$ Department of Chemistry and Biochemistry, Kent State University, Kent, OH 44242, USA. ${ }^{2}$ Biomolecular Nanoscale Engineering Center, Department of Physics, Chemistry and Pharmacy, University of Southern Denmark, Campusvej 55, 5230 Odense M, Denmark. ${ }^{3}$ ATDBio Ltd., Magdalen Centre, Oxford Science Park, 1 Robert Robinson Avenue, Oxford OX4 4GA, UK. ${ }^{4}$ Department of Chemistry, University of Copenhagen, Thorvaldsensvej 40, 1871 Frederiksberg, Denmark. ${ }^{5}$ Department of Chemistry, University of Copenhagen, Universitetsparken 5, 2100 Copenhagen, Denmark. ${ }^{6}$ Department of Chemistry, University of Oxford, 12 Mansfield Road, Oxford OX1 3TA, UK. ${ }^{7}$ These authors contributed equally: Shankar Pandey, Shankar Mandal, Mathias

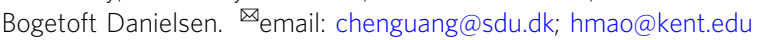


C hiral recognition is omnipresent in asymmetric reactions biased toward one of the two stereoisomers ${ }^{1-3}$. Beyond small molecules, chiral communications have been reported in secondary structures of foldamers and copolymers via a network of non-covalent interactions ${ }^{4-7}$. In copolymers, various modes of chiral communications have been found ${ }^{8,9}$. For foldamers and non-proteogenic peptides, chirality has been induced by different chiral modalities ${ }^{10-18}$, while screw-sense reversal has been observed ${ }^{19-21}$. In natural biomacromolecules, helical sense mismatch phenomena were noticed in the $\mathrm{B}-\mathrm{Z}$ transition region of DNA double helices where the two opposite helical senses encountered, breaking one base pair while perturbing two adjacent bases to accommodate the torsional strain ${ }^{22}$.

However, all chiral communications demand close contact of neighbouring functional groups, including chiral inducers. In the contact interface, the interaction strength is determined by an ensemble set of intermolecular forces (IMF) between functional groups, which is effective on the length scale of Van der Waals radius $^{23}$. Therefore, it remains elusive whether chiral-to-chiral communication is permitted beyond the IMF distance between higher-order macromolecular domains such as peptide tertiary structures. As one of the main protein tertiary structures 24,25 , peptide coiled-coil domains compose of multiple a-helices wrapped around one another to generate multimeric right- or left-handed helical structures ${ }^{26}$. The inter-strand helicity of these domains are mechanical in nature 27 . It is thus tantalizing to see whether chirality can be transmitted by mechanical interactions, which are long-range in nature.

Compared to DNA helices, the peptide coiled-coil helices have much less inter-strand twisting in which $\alpha$-helices wrap around one another ${ }^{28,29}$. As a result, the chiral communication between the inter-strand helical senses of two neighbouring protein coiled coils is difficult to detect by ensemble average approaches with low chiral sensitivities. Here we propose to employ singlemolecule force spectroscopy to investigate whether chiral-tochiral communication is permitted from enantiomeric trimeric peptide coiled coils to a DNA triplex (Fig. 1). The DNA triplex, composed of a triplex-forming oligonucleotide (TFO) binding to the major groove of a duplex DNA, has shown to facilitate peptide coiled-coil self-assembly ${ }^{30-32}$. To achieve this templating effect, each peptide strand was conjugated with a DNA strand, resulting in a peptide-oligonucleotide-conjugate (POC) in which the length of the interdomain linker can be varied. The chiral transmission was evaluated by the binding efficacy of the third POC to the underlying POC duplex.

Overall, we observed facile binding of the third POC strand to the POC duplex system, likely due to the templated binding effect in which conjugated peptide-oligonucleotide geometry significantly increased the effective concentration of either peptide or oligonucleotide. The sandwiched triple-stranded linker region (24-25 bonds) was previously found to be packed tightly for both L-peptide/D-DNA and D-peptide/LDNA triple helices ${ }^{30}$, giving rise to a torsionally constrained intersection between two macromolecular domains. In analogy to the helical sense mismatch in the B-Z DNA junctions, when the two torsionally constrained, covalently conjugated macromolecular domains adopt opposite inter-strand helical senses, they may result in a less stable macromolecular state (Fig. 1, as exemplified by a hybrid DNA-peptide triple helix structure shown at the left in the inset). Relative to the case of helical sense match where DNA strands and peptide coiled coils have the same inter-strand helical sense (Fig. 1, right in the inset), this reduced stability would be manifested in compromised binding of the third POC strand to the POC duplex templates, which was confirmed by experiments. Small-angle X-ray scattering (SAXS) and molecular dynamics (MD) simulation revealed no direct electrostatic/hydrophobic contact between the two macromolecular domains. Instead, the three interdomain linkers adopted restricted conformations via hydrophobic interactions, which likely explained the chiral conduction between the trimeric peptide coiled-coil and the DNA triplex. These findings indicated that chiral communications are not only present in the secondary structures of copolymers and non-proteogenic peptides, but also exist between two biomacromolecular domains in a long-range manner $(<4.5 \mathrm{~nm})$, favouring the homochirality of neighbouring peptide coiled-coil domains in proteins.

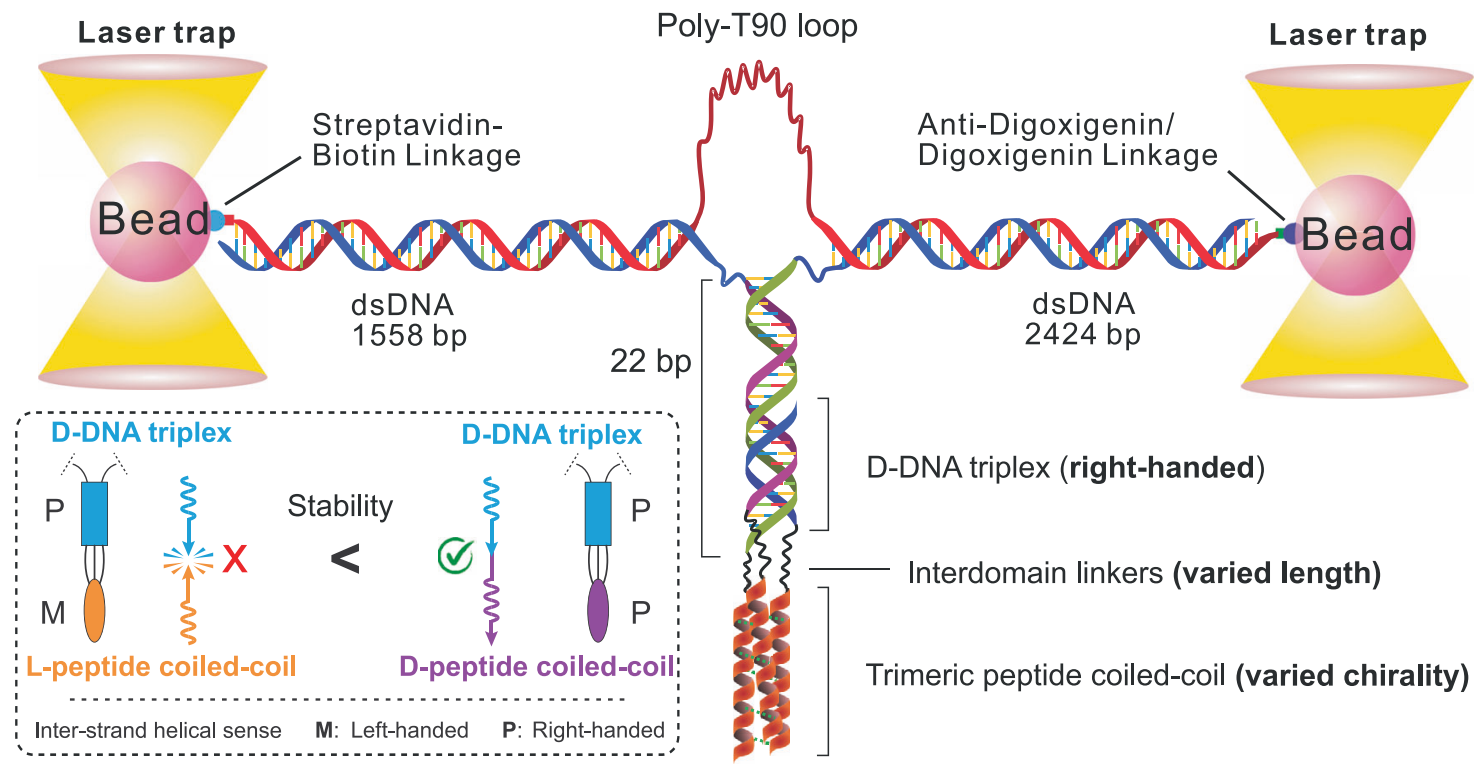

Fig. 1 Schematic of optical-tweezers set up for high-throughput single-molecule assay. The inter-strand helical sense match/mismatch effects between the two macromolecular domains are shown in the bottom left inset in which the D-DNA triplex is marked in cyan rectangle, L-peptide coiled-coil in orange ellipse and D-peptide coiled-coil in purple ellipse. M and P stand for left-handed and right-handed inter-strand helical senses, respectively. The clash symbol depicts the helical sense mismatch state, which has unfavourable energy cost and thus is marked by a red-cross. The no clash symbol represents the helical sense match state, which should not influence the stability and thus is marked by a green check. 
A)

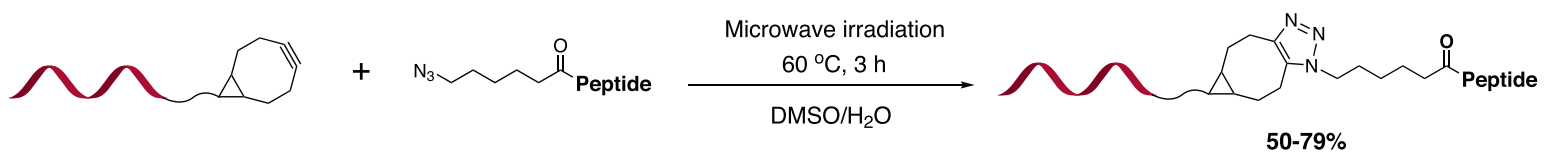

ON1-18-BCN

Azidopeptides

L,D-POC1-18 and D,D-POC1-15

B)

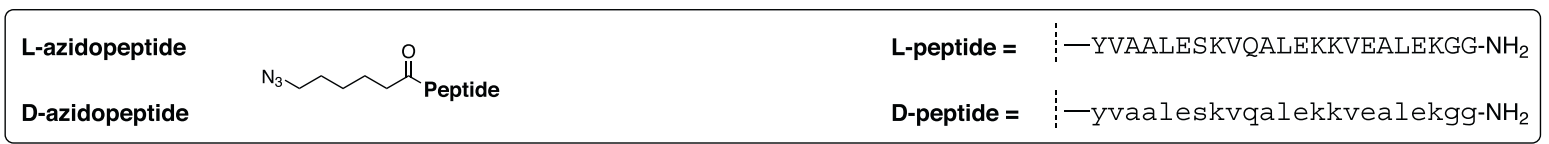

Triplex

ON $(1,4,7,10,13,16)$-BCN

ON $(2,5,8,11,14,17)-B C N$

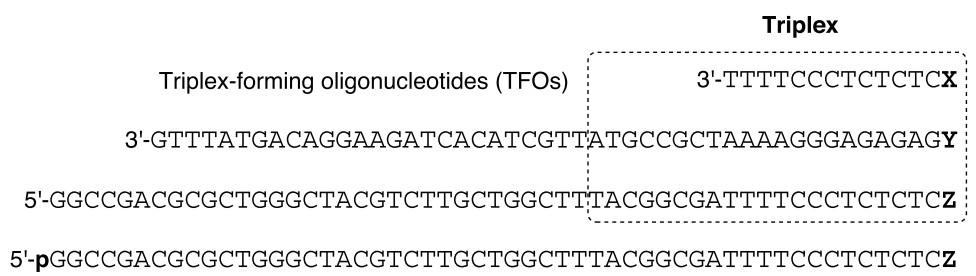

ON $(3,6,12,15,18)-B C N$

5'-pGGCCGACGCGCTGGGCTACGTCTTGCTGGCTTTACGGCGATTTTCCCTCTCTCZ

ON9-BCN $\quad \mathbf{p}=$ phosphate group
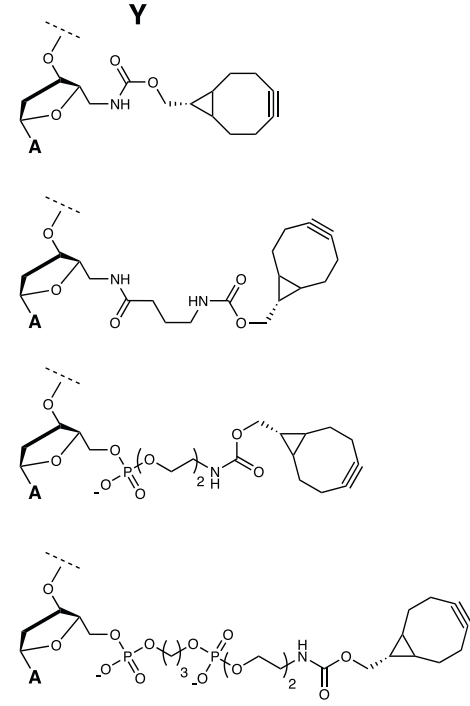

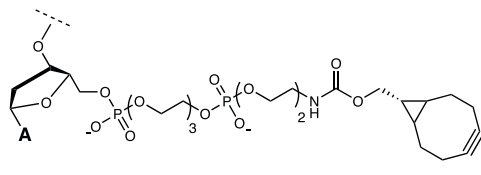<smiles>O=C(NCCOP(=O)(O)OCCOP(=O)(O)OC1CC2CCC1C2)OCC1CC=CCC1</smiles><smiles>CCOC1CCOC1COP(=O)(O)OCCOP(=O)(O)OCCNC(=O)OCC1CCC=CCC1</smiles>
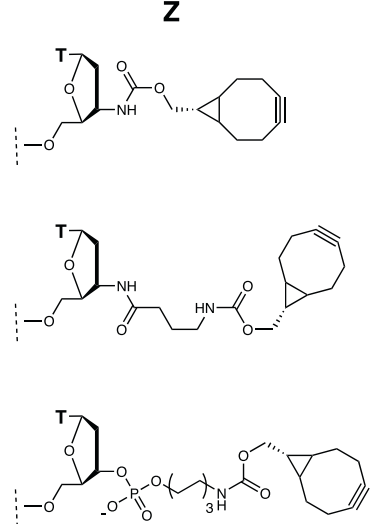<smiles>COCCOC(=O)NCCOP(=O)(O)OCCOC(=O)OC1CC2CCCCC2C1</smiles>

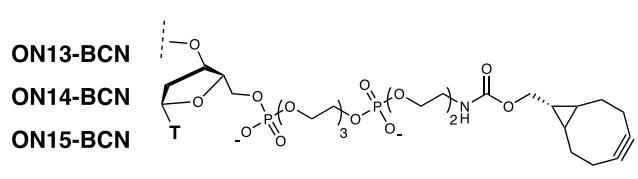

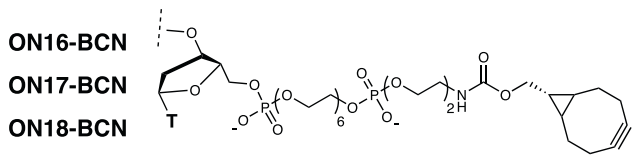

Fig. 2 POC synthesis. A Conjugating two stereoisomeric azidopeptides to ON-BCNs via strain-promoted alkyne-azide cycloaddition to obtain L,D-POC1-18 and D,D-POC1-15. B Sequence of L-azidopeptide, D-azidopeptide and ONs. C Different linker lengths were employed to furnish the BCN function either on the $5^{\prime}$-end or on the $3^{\prime}$-end of ONs. Natural amino acids are denoted in upper cases while unnatural ones are in lower cases. Thus, Y, V, L, E, S, K, Q, A, G are L-amino acids whereas $y, v, l, e, s, k, q, a, g$ are D-amino acids. A, G, C, and T are natural DNA monomers. Key: The two-letter prefix before POCs: The first letter indicates the chirality of the peptide while the second letter indicates the chirality of the ON.

\section{Results}

Design and synthesis of POC. DNA helices were chosen as the first set of macromolecular helical domain, while two mirrorimaged coiled-coil peptide tertiary structures ${ }^{30}$ were employed as another set of macromolecular helical domains derived from coil$\mathrm{V}_{\mathrm{a}} \mathrm{L}_{\mathrm{d}}{ }^{33}$. A series of linker lengths were introduced between the oligonucleotide triplex and the peptide tertiary structures, including 16 bonds, 21 bonds, 24-25 bonds, 30-31 bonds, 35-36 bonds and 44-45 bonds, each counting as the shortest path from $5^{\prime}-/ 3^{\prime}$-position $\left(5^{\prime}-\mathrm{NH} / 3^{\prime}-\mathrm{NH}\right.$ or $\left.5^{\prime}-\mathrm{O} / 3^{\prime}-\mathrm{O}\right)$ of the oligonucleotide (ON) triplex to the N-termini ( $\mathrm{NH}$ of $\mathrm{Tyr}$ ) of the peptide (Fig. 2).

The two peptides, L-azidopeptide and D-azidopeptide, were synthesized through solid-phase peptide synthesis ${ }^{30}$. For the ON triplex, automated DNA synthesis were carried out for eighteen ONs (see Supplementary Discussion). Phosphoramidite monomers 4, 13, 7, 16 (Supplementary Figs. 1 and 2) and four commercially available monomers were incorporated to the $5^{\prime}$-end of ONs (ON1, ON2, 
ON4, ON5, ON7, ON8, ON10, ON11, ON13, ON14, ON16 and ON17, Supplementary Fig. 10), while the synthesis of ON3, ON6, ON9, ON12, ON15 and ON18 (Supplementary Fig. 10) started from three special solid supports (3'-amino-dT CPG, 23 in Supplementary Fig. 3 and $3^{\prime}$-PT-amino-modifier C6 PS). After reverse-phase and/or ion-exchange HPLC purification, the composition and purity $(>95 \%)$ of eighteen ONs were confirmed by ionexchange HPLC, MALDI-MS and ESI-MS (Supplementary Figs. 4-9). A two-step synthetic strategy was used to prepare the POCs (Fig. 2 and Supplementary Figs. 10-44). A total of 33 POCs (Supplementary Fig. 45) were synthesized to assemble eleven designer POC triplexes with the interdomain linker length increasing from 16 bonds (L,D-POC $(\mathbf{1}+\mathbf{2}+\mathbf{3})$, D,D-POC $(\mathbf{1}+\mathbf{2}+\mathbf{3}))$, 21 bonds (L,D-POC $(\mathbf{4}+\mathbf{5}+\mathbf{6})$, D,D-POC $(\mathbf{4}+\mathbf{5}+\mathbf{6})$ ), $24-25$ bonds (L,D-POC $(7+8+9)$, D,D-POC $(7+8+9))$, 30-31 bonds (L,DPOC $(\mathbf{1 0}+\mathbf{1 1}+\mathbf{1 2})$, D,D-POC $(\mathbf{1 0}+\mathbf{1 1}+\mathbf{1 2})), 35-36$ bonds (L,DPOC $(13+14+15)$, D,D-POC $(13+14+15))$ and $44-45$ bonds $($ L,D-POC $(\mathbf{1 6}+\mathbf{1 7}+\mathbf{1 8}))$. Only L,D-POCs were synthesized for the longest linker with $44-45$ bonds. The right-handed helical sense was always maintained for the DNA triplex, while the inter-strand screw sense of coiled coils varied from left-handedness to right-handedness when L-peptide coiled-coil was replaced with D-peptide coiled-coil (Fig. 1).

Formation of trimeric coiled-coil peptides increases the mechanical stability of DNA triplex templates. We used a single-molecule mechanical platform in optical tweezers to investigate the repetitive formation and dissociation of D-peptide or L-peptide coiled coils assisted by the DNA templates (Supplementary Fig. 46). Each single-stranded DNA fragment of the assembled dimeric POC assembles was hybridized with a complementary DNA overhang at the end of a duplex DNA handle. The free end of one DNA handle was labelled with digoxigenin, while that of the other DNA handle was labelled with biotin. The digoxigenin and biotin ends were then bound to two optically trapped beads coated with digoxigenin antibody and streptavidin, respectively (Fig. 1). A polythymine (T90) linker was used to connect the two DNA handles from the non-labelled ends, so that the tethered POC strands can be kept in proximity ${ }^{34}$ to each other. This facilitated the reassembly of the POC complexes at the reduced force after they were disrupted mechanically at higher force.

Two POC fragments were brought closer with the help of a steerable mirror, which moved one of the optically trapped beads with respect to the other. The hybridized duplex DNA domain served as a template to assemble dimeric coiled-coil peptides in the POC duplex. Since acidic condition is required to form stable POC triple helices (protonation of $\mathrm{N} 3$ of cytosine to provide an extra $\mathrm{H}$-bond in the $\mathrm{ON}$ triplex $\left.{ }^{35,36}\right), \mathrm{pH} 5.5$ was maintained throughout all the experiments. By moving optically trapped beads away from each other, the tension accumulated in the molecular construct was solely exerted on the dimeric POC complex. The increased tension eventually dissociated the two POC strands, which was recorded in real-time in the forceextension curves (Fig. 3B, middle). Using the DNA duplex as the control, experiments were firstly carried out for two dimeric POC complexes, D,D-POC $(2+3)$ and L,D-POC $(2+3)$, all of which uniformly gave a rupture force at $\sim 14 \mathrm{pN}$ (Fig. 3A-C). Thus, dimeric POC complexes did not yield differentiating signals to probe the proposed long-range chirality effect on the topology of higher-order macromolecular structures. The mechanical stability of the dimeric L,D-POC $(2+3)$ complex $(13.8 \mathrm{pN}$, Fig. 3C) was found to be slightly lower than the corresponding D,D-POC $(2+3)$ complex (14.4 pN, Fig. 3B).
We then flowed the TFO/D,D-POC1 $(1 \mu \mathrm{M})$ into the chamber at the same $\mathrm{pH}$ (Fig. 1A, bottom), which resulted in the formation of the triplex by the Watson-Crick and Hoogsteen base-pairing (Fig. 3D) 37,38 . When TFO (Supplementary Fig. 47) bound to the single-molecule DNA duplex target, mechanical unfolding revealed that the $13.6 \mathrm{pN}$ as the unzipping force for the DNA triplex, indicating a weaker apparent mechanical stability compared to the DNA duplex (14.6 pN, Fig. 3A), which was consistent with literature observation ${ }^{39}$. The reduced mechanical stability was interpreted as a minority of DNA duplex formation being perturbed as the result of the TFO injection. In the next experiment, we replaced the DNA triplex template with three POC strands to assemble the chimeric POC triplex where the formation of the DNA triple helix, and the trimeric peptide coiled-coil were cooperative ${ }^{30}$. This chimeric supramolecular complex D,D-POC $(\mathbf{1}+\mathbf{2}+\mathbf{3})$ showed much stronger mechanical stability $(23.2 \mathrm{pN}$, Fig. 3E), indicating that the synergy between the peptide domain and the nucleic acid template significantly improved the mechanical stability of the underlying DNA triplex scaffold. These results validate that trimeric POC complexes may serve as ideal macromolecular tools to determine the helical sense mismatch effect for macromolecular topologies since the synergistic formation of the chimeric POC triplex can be unequivocally distinguished by the $23 \mathrm{pN}$ unfolding force populations.

Long-range chirality transmission between natural nucleic acid domain and peptide domain in a distance-dependent manner. When we compared the $23 \mathrm{pN}$ population between the two D,D$\operatorname{POC}(\mathbf{1}+\mathbf{2}+\mathbf{3})$ and L,D-POC $(\mathbf{1}+\mathbf{2}+\mathbf{3})$ trimeric coiled-coil diastereomers, the latter species showed a lower formation percentage ( $98 \%$ vs $87 \%$, at $99.99 \%$ confidence level, Fig. $4 \mathrm{~A})$. Given that the DNA triplex and the D-peptide coiled-coil shared the same inter-strand screw sense, whereas the DNA triplex and L-peptide coiled-coil had the opposite helicities, we reasoned that the inter-strand screw-sense mismatch in the DNA-peptide interface may disfavour the synergistic formation of the chimeric POC triplex. The lower force populations $(13 \mathrm{pN}, 13 \%)$ for L,D-POC $(\mathbf{1}+\mathbf{2}+\mathbf{3})$ were presumed to originate from the dissociation of the POC duplex, where there was no binding for L,DPOC1. These observations encouraged us to pursue more confirmative evidence for the chirality communication between two macromolecular domains.

Thus, as a continued step we varied the length of the linker bridging the DNA and the peptide domains, which was extended to 21 bonds (L,D-POC $(\mathbf{4}+\mathbf{5}+\mathbf{6})$, D,D-POC $(\mathbf{4}+\mathbf{5}+\mathbf{6})$ ), $24-25$ bonds (L,D-POC $(7+8+9)$, D,D-POC $(7+8+9)), 30-31$ bonds $(\mathrm{L}, \mathrm{D}-\mathrm{POC}(\mathbf{1 0}+\mathbf{1 1}+\mathbf{1 2}), \quad \mathrm{D}, \mathrm{D}-\mathrm{POC}(\mathbf{1 0}+\mathbf{1 1}+\mathbf{1 2})), \quad 35-36$ bonds (L,D-POC $(13+14+15)$, D,D-POC $(13+14+15))$ and 44-45 bonds (L,D-POC $(\mathbf{1 6}+\mathbf{1 7}+\mathbf{1 8})$ ) (Supplementary Fig. 45). For each linker, we determined the unfolding force and percentage formation of the chimeric POC triplex (Fig. 4A, B). Two general trends were observed: (1) The mechanical stability of either loweror higher- force population in homochiral D-peptide/D-DNA complexes always appeared higher than the corresponding species in heterochiral L-peptide/D-DNA complexes, congruent with the results obtained from L,D-POC $(2+3)$ and D,D-POC $(2+3)$, as well as from L,D-POC $(\mathbf{1}+\mathbf{2}+\mathbf{3})$ and D,D-POC $(\mathbf{1}+\mathbf{2}+\mathbf{3})$. While the DNA triple helix held the same right-handedness, considering the opposite mechanical chirality in the two mirrorimaged trimeric peptide coiled coils, these results clearly supported our hypothesis that the unmatched inter-strand screw-sense reduced the mechanical stability of the dimeric and trimeric POC assemblies. (2) By comparing the population percentage of the POC triple helix, the homochiral complexes 
A
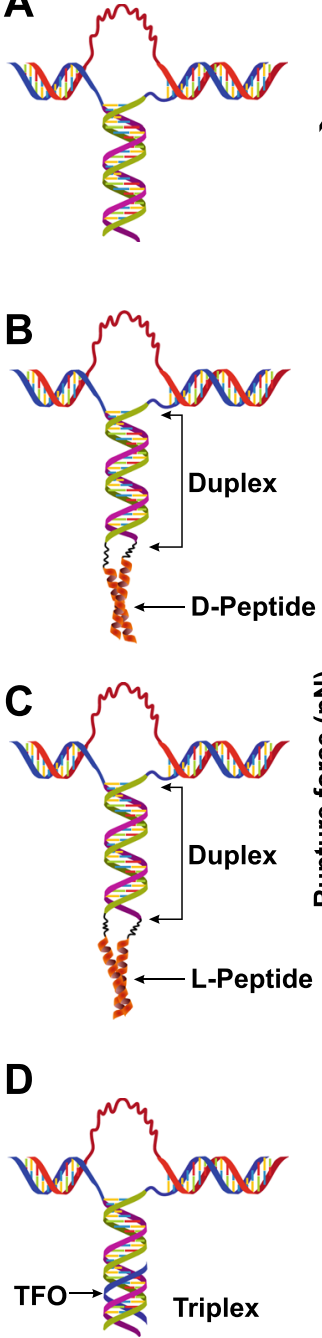

E

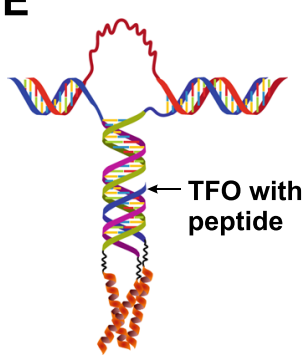

Duplex without peptide
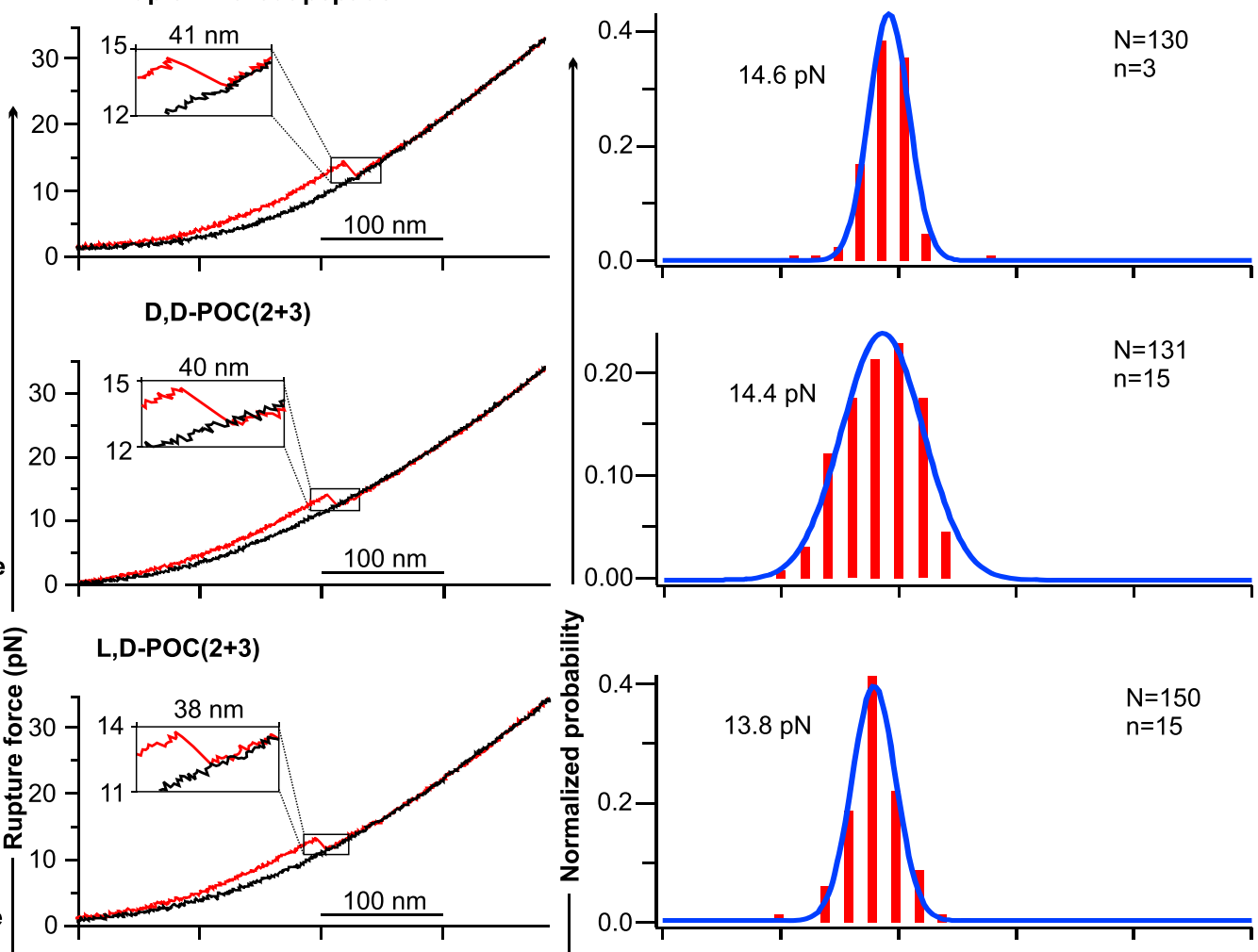

TFO without peptide

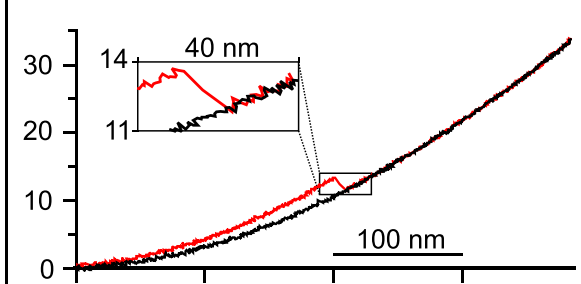

D,D-POC $(1+2+3)$

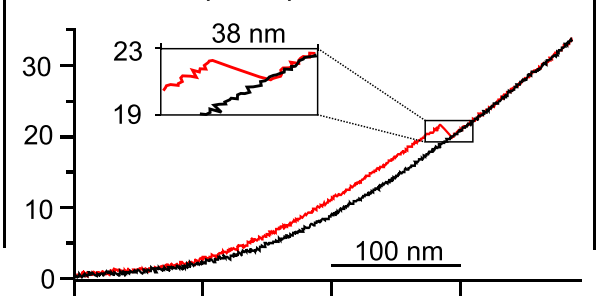

Extension (nm)
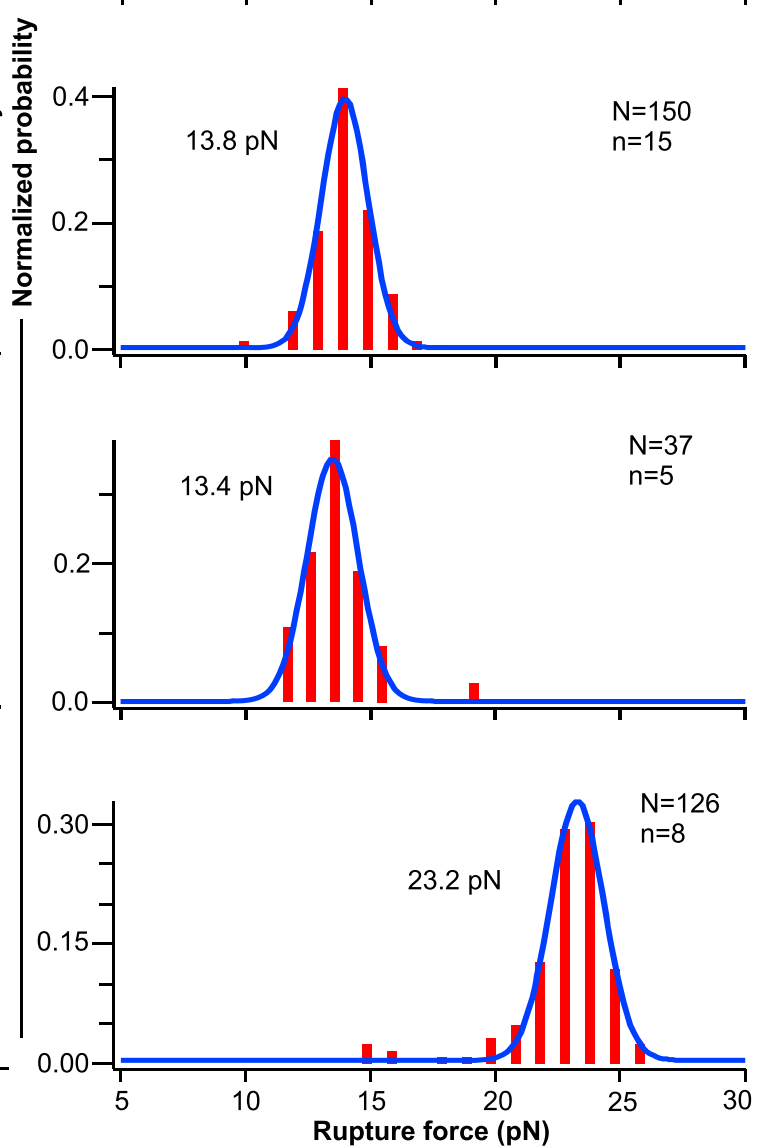

Fig. 3 Effect of TFO or D,D-POC1 on the mechanical stability of the DNA duplex or the chimeric POC duplex D,D-POC(2+3). Schematic diagrams, typical force-extension curves and corresponding force histograms of (A) DNA duplex control, (B) D,D-POC(2 + 3), (C) L,D-POC(2 + 3), (D) DNA triplex control, (E) D,D-POC(1 $+\mathbf{2}+\mathbf{3})$. TFO = 3'-TTTTCCCTCTCTCT. Note: red and black traces in a force-extension curve represent stretching and relaxing events, respectively. $N$ and $n$ depict total numbers of features and distinct molecules, respectively.

(D-peptides and D-DNA) were more favoured than the heterochiral complexes (L-peptide and D-DNA) at each linker length. This further validated the presence of the postulated remote chirality transmission between the employed nucleic acid and peptide structures.

Interestingly, when we plot out the populations of the trimeric coiled coils vs linker length (Fig. 4B), we found that the Dpeptide/D-DNA system had a monotonic decrease in population, whereas the L-peptide/D-DNA had a minimum triplex peptide population when the linker length was $24-25$ bonds in
L,D-(POC7 + POC8 + POC9). For the D-peptide/D-DNA system, the decrease in the chimeric triplex population over the linker distance was probably due to the weakened templated binding effect (which gradually reduced when the interdomain linker was elongated). In marked contrast, for the L-peptide/DDNA, the templated binding effect and the helical sense mismatch effect had the opposite momentum, thus resulting in a fluctuated population landscape for the chimeric triple helix. An exceptional example was when the linker became relatively short (16 bonds in L,D-POC $(\mathbf{1}+\mathbf{2}+\mathbf{3})$ and D,D-POC $(\mathbf{1}+\mathbf{2}+\mathbf{3})$ ), 

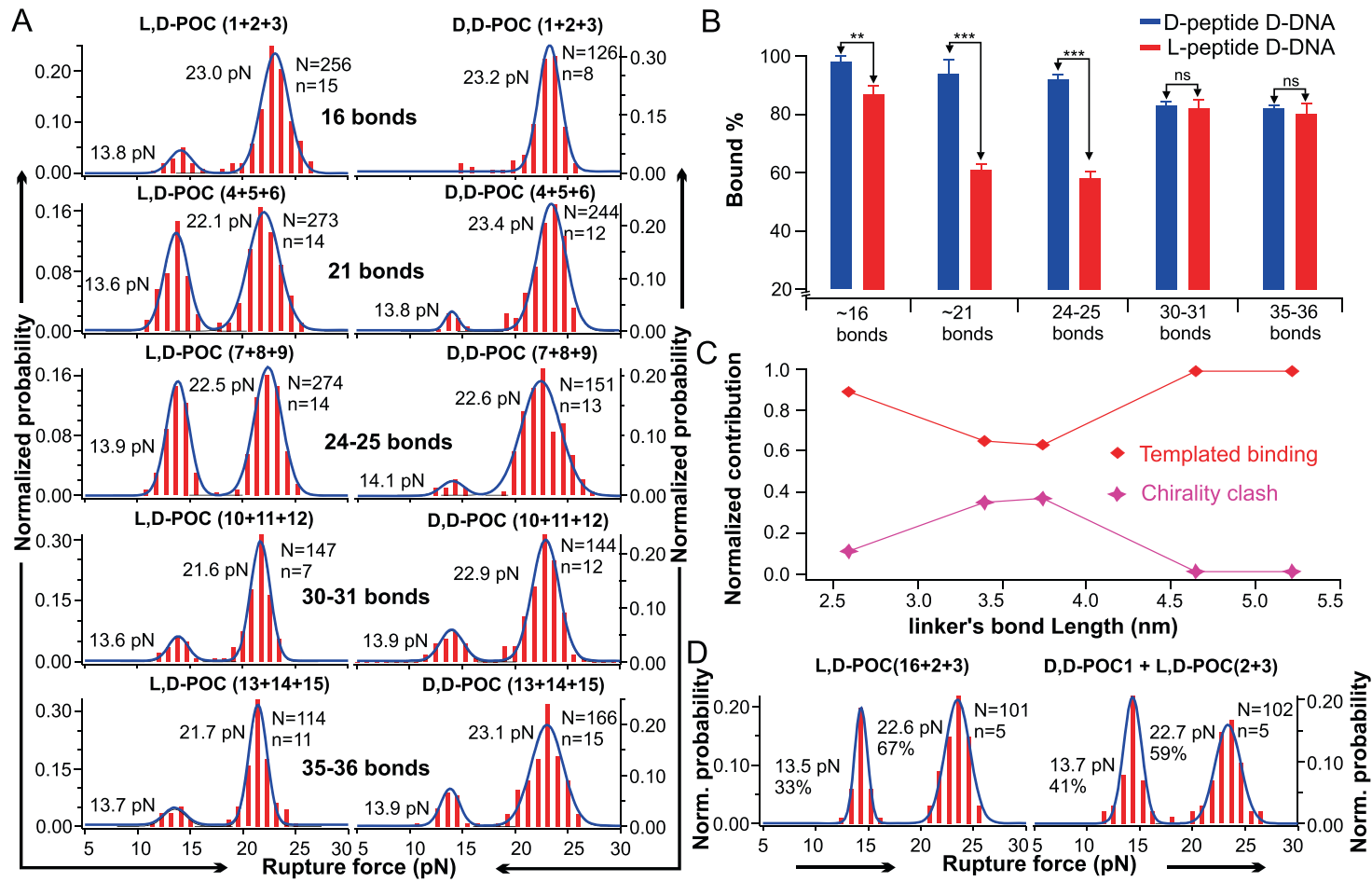

Fig. 4 Comparative study of bound \% for the L- and D-peptide with different linker lengths. A Rupture force histograms for the L-peptide D-DNA (left) and D-peptide D-DNA (right) with varying linker length (in number of bonds). B Comparison of bound \% for D-peptide D-DNA and L-peptide D-DNA. Data are presented as mean \pm SD for $n=4$ independent experiments. ${ }^{\star \star} P=0.0085,{ }^{\star \star \star} P=3.9 \times 10^{-5}$ for the 21 bonds and $P=2.4 \times 10^{-5}$ for the $24-25$ bonds, ns: nonsignificant (two tailed unpaired $t$-test). C Percentage contribution of chirality clash and templated binding effects for the trimeric L-peptide D-DNA with varied linker length (see Methods for calculation). D Rupture force histograms for the L-peptide D-DNA (L,D-POC(2 + 3), 16 bonds) bound with a TFO conjugated with L-peptide (L,D-POC16, 44-45 bonds, left) or with D-peptide (D,D-POC1, 16 bonds, right). $N$ and $n$ depict total numbers of features and distinct molecules, respectively.

due to the predominating templated binding effect, high chimeric trimer populations were observed in both cases irrespective of $\mathrm{D}$ or L-peptide. When the interdomain linker was elongated from 16 bonds $(2.6 \mathrm{~nm})$ to 21 bonds $(3.4 \mathrm{~nm})$, the helical sense mismatch effect became significantly more pronounced. When it was further extended to $24-25$ bonds $(3.7 \mathrm{~nm})$, the helical sense mismatch effect culminated, inhibiting the chimeric triplex population to a minimum level for the L-peptide/D-DNA system. This trend confirmed the long-range chirality transmission between the two macromolecular domains through three long interdomain linkers. Though each linker has more than half of the bonds with rotational freedom, we envisioned that packing three linkers between the two macromolecular domains may convert them to a superhelical conductor ${ }^{22}$ by forcing them to adopt restricted conformation(s) (vide infra). At even longer linker length (30-31 bonds and beyond), the helical sense mismatch effect sharply faded away close to zero level.

To quantify the relative contribution of the templated binding effect and the helical sense mismatch effect, we determined the percentage of the helical sense mismatch contribution for Lpeptide/D-DNA (Fig. 4C). Assuming there was no helical sense mismatch effect in the D-peptide/D-DNA system, the maximum templated binding effect (100\%) was determined by the percentage population of the D-peptide/D-DNA triplex at each linker length. On the other hand, when there was $100 \%$ helical sense mismatch, the L-peptide/D-DNA triplex population would be reduced to zero. By comparing the percentage formation of the two diastereoisomeric POC triplexes against these two extreme conditions (see Methods section), the percentage contribution of the helical sense mismatch effect for the L-peptide/D-DNA was determined at each linker length (Fig. 4C). From this analysis, a clear trend was noticed that the helical sense mismatch effect was long-range, reaching a maximum at $3.7 \mathrm{~nm}$ (contour length) before quickly diminishing to zero beyond $4.5 \mathrm{~nm}$ (contour length). The templated binding effect was somehow more profound. For instance, at $6.6 \mathrm{~nm}$ (linker with $44-45$ bonds, Supplementary Fig. 45) the templated binding effect still persisted to facilitate the formation of the trimeric coiled coils (L,DPOC $(16+17+18)$, Supplementary Fig. 48A). All investigated POC duplexes/triplexes gave almost identical values in change-incontour-length (Supplementary Fig. 49), confirming that the unfolding of POC triplexes took place when the underlying POC duplexes were ruptured.

To provide further evidence for the helical sense mismatch effect and the templated binding effect, we varied the interdomain linker length as well as the chirality of the peptide in the third POC, which was added through microflow injection. First, we used L,D-POC $(2+3)$ (linker length, 16 bonds) and flowed L,DPOC16 (linker length, 44-45 bonds). We observed that the percentage formation of the homochiral chimeric triplex was decreased to $67 \%$ (Fig. $4 \mathrm{D}$, compared to $87 \%$ in Fig. $4 \mathrm{~A}$, top left panel). Similarly, when we tested the binding of L,D-POC(17+ 18) (linker length, $44-45$ bonds) in the presence of L,D-POC1 (linker length, 16 bonds), we again observed decreased homochiral triplex formation (from $80 \%$ [L,D-POC $(\mathbf{1 6}+\mathbf{1 7}+\mathbf{1 8})]$ to $52 \%$ [L,D-POC $(\mathbf{1}+\mathbf{1 7}+\mathbf{1 8})]$ (Supplementary Fig. 48). In both cases, the inter-strand screw-sense mismatch was preserved between the DNA triplex and the L-peptide coiled-coil trimer. Thus, the reduced triplex formation could be ascribed to the entropic penalty that originated from mismatched linker lengths 
between 16 bonds and 44-45 bonds. Therefore, these two experiments proved that the templated binding effect mediated by the same or similar linker length is important for the trimeric coiled-coil formation. In another control, we investigated the binding of D,D-POC1 (linker length, 16 bonds) to the L,DPOC $(2+3)$ (linker length, 16 bonds) single-molecule target, both of which had the same linker length but opposite chirality in the peptide domain. We found that the percentage formation of the trimeric POC ensemble was decreased to 59\% (Fig. $4 \mathrm{D}$, compared to $87 \%$ in Fig. 4A, top left panel). Such a result demonstrated that a perfect chirality match in different domains was indispensable to achieve the desired stabilizing synergy between the trimeric peptide coiled-coil and the DNA triplex scaffold. Finally, to explore the possible kinetic influence, we performed the single-molecule unfolding experiment for the L,DPOC system with $24-25$ bonds linker length by decreasing the time window for POC triplex formation (incubation time at $0 \mathrm{pN}$ after each unfolding event) from 60 to $15 \mathrm{~s}$ (Supplementary Fig. 50). We found percent formation of L,D-POC $(7+8+9)$ trimeric complex was $61 \%$, which was very close to the $58 \%$ formation at 60 -second incubation time, indicating binding of the third POC strand reached equilibrium in our single-molecule experiments.

CD spectra were also recorded on all eleven POC triplex assemblies with the two ssDNA regions fully paired with their DNA complements. After subtracting the signatures contributed by the ON triplex (a DNA triplex control was used) to isolate the signal from the peptide structures, strong $\alpha$-helicity was observed for all eleven POC triplex ensembles (both L-peptide and Dpeptide, Supplementary Fig. 51), indicating that the formation of the coiled-coil structure was not significantly perturbed when the interdomain linker was elongated from 16 to $44-45$ bonds. These experiments supported the premise that high force populations in mechanical unfolding were indeed from trimeric POC assemblies.

In addition, non-denaturing polyacrylamide gel electrophoresis was carried out for $15 \mu \mathrm{M}$ of TFO alone, L,D-POC7 and D,DPOC7, to eliminate the possibility that different intramolecular/ intermolecular complex formations of the third POC strands accounted for the obtained single-molecule experimental results (Supplementary Fig. 52). Both L,D-POC7 and D,D-POC7 gave their bands near the 35-mer DNA marker, located very close to the monomeric state of the L,D-POC $(\mathbf{8}+\mathbf{9}) * 31$ but far away from the TFO alone control. This indicated that both L,D-POC7 and D,D-POC7 likely adopted fully extended conformations. Self-dimerization close to 75-mer DNA marker was also noticed ( $\sim 8 \%$ for D,D-POC7 and $<5 \%$ for L,D-POC7, quantified in ImageJ), probably via intermolecular peptide interactions. However, considering the lower concentration $(1 \mu \mathrm{M})$ used in the single-molecule experiments, which disfavoured the intermolecular interactions, we expect intermolecular interaction of the third POC strands had little influence on the bound fraction of the POC triplexes for L,D-POC $(7+8+9)$ and D,D-POC $(7+8+9)$.

SAXS analysis and MD simulations. To investigate the molecular mechanism of the chiral transmission between trimeric peptide coiled coils and DNA triplex, we performed SAXS and molecular modelling studies on the diastereoisomeric pair of D,D-POC $(7+8+9)$ and L,D-POC $(7+\mathbf{8}+\mathbf{9})$ since they represent the highest change in the triplex population ( $96 \%$ vs $58 \%$ ). To ease the experimental work, the two ssDNA regions extended from the underlying POC duplex were truncated, leading to the corresponding two diastereoisomers D,D$\operatorname{POC}(7+8+9)^{*}$ and L,D-POC $(7+8+9)^{*} \quad$ (Supplementary Fig. 53). The SAXS characterization of D,D-POC $(7+8+9)^{*}$ (Supplementary Fig. 54) showed the signals strongly resembled its diastereoisomer L,D-POC $(\mathbf{7}+\mathbf{8}+\mathbf{9})^{*}$ (Supplementary Fig. 55) ${ }^{30}$, suggesting formation of well-defined structures. The long-tailed realspace distance distribution function shown in Supplementary Fig. 54c confirmed that the conformation(s) of D,D-POC $(7+\mathbf{8}+\mathbf{9})^{*}$ were extended in the given buffer solution ${ }^{40}$. MD simulations indeed demonstrated more distinct and compact conformations after $\sim 100$ ns compared to the extended initial structure (Supplementary Figs. 56 and 57). While no direct electrostatic/hydrophobic contact between the DNA triplex and the trimeric peptide coiled-coil was detected in the MD ensembles (Supplementary Movie 1), the best SAXS fit from a single MD snapshot (representing a significantly populated group of compact conformations) depicted a bend in the linker region (Supplementary Fig. 58).

The single MD snapshot containing representative features of the entire $\mathbf{D}, \mathbf{D}-\mathbf{P O C}(\mathbf{7}+\mathbf{8}+\mathbf{9})^{*}$ further showed that the linkers packed against each other and against the oligonucleotide and peptide termini (Fig. 5a-c). These interactions were formed during the first half of the MD simulation and caused the simulation structure to bend and become more compact with time. In the last half of the simulation, an intramolecular stacking interaction persisted between a linker triazole moiety and a terminal thymine, as illustrated in Fig. 5a, b. The two other linkers associated closely with each other, as seen from the side (Fig. 5a) and top views along the coiled-coil axis (Fig. 5C). Similar interactions were present in the MD simulation of $\mathbf{L}, \mathbf{D}$ $\operatorname{POC}(7+8+9)^{* 30}$ (Fig. $5 \mathrm{~d}-\mathrm{f}$ ), where two linkers associated by hydrophobic interactions, while the third linker was transiently separated from the two other linkers by the insertion of a tyrosine side chain (white arrow in Fig. 5d, f). Transient hydrogen bonds were also noticed between the N-terminal tyrosine phenolic $\mathrm{OH}$ groups and terminal oligonucleotide backbone phosphates. Overall, the linker regions of both $\mathrm{D}, \mathrm{D}-\operatorname{POC}(\mathbf{7}+\mathbf{8}+\mathbf{9})^{*}$ and its diastereomer L,D-POC $(7+\mathbf{8}+\mathbf{9}) * 30$ became compact due to the linker-nucleotide stacking, hydrophobic linker-linker interactions and linker-tyrosine interactions. Such a compact linker region limits the rotational freedom of individual linkers, allowing chiral communication between the DNA triplex and the trimeric peptide coiled-coil.

\section{Discussion}

All obtained results coherently supported our hypothesis that the chirality of supercoils can be transmitted between peptide coiled coils and DNA helices in a long-range manner. From SAXS and MD simulation, it is clear that no direct interaction exists between the trimeric peptide coiled-coil and DNA triplex domains. In addition, the three linkers have shown constrained conformations packed between two domains. Therefore, the chiral communication is very likely transmitted through these spatially constrained linkers in a manner similar to the transfer of superhelicity in torsionally constrained duplex DNA templates ${ }^{41}$. To the best of our knowledge, this is the first example of a chiral-to-chiral communication propagated between two biomacromolecular domains (Fig. 6). Our findings may explain the observation that opposite inter-strand helical senses rarely exist in proximity inside the same macromolecule due to unfavourable energy cost ${ }^{22}$.

Natural peptides can either assume left-handed or right-handed helices, which intertwine one another to form right-handed ${ }^{42,43}$ or left-handed screw sense $\mathrm{s}^{44-49}$, respectively. Intriguingly, opposite coiled-coil helicities are only observed in proteins with the aid of either transition motifs (stutters) and/or the binding of other proteins $s^{50}$. For example, one research study demonstrated that a left-handed and a right-handed trimeric coiled-coil could simultaneously exist in the crystal structure of an immunoglobulin-binding protein D from Escherichia coli, where a 22-mer antiparallel $\beta$-sheet saddle $(\mathrm{ca} .15 \mathrm{~nm})$ was inserted in $\sim 120^{\circ}$ rotation between the two 

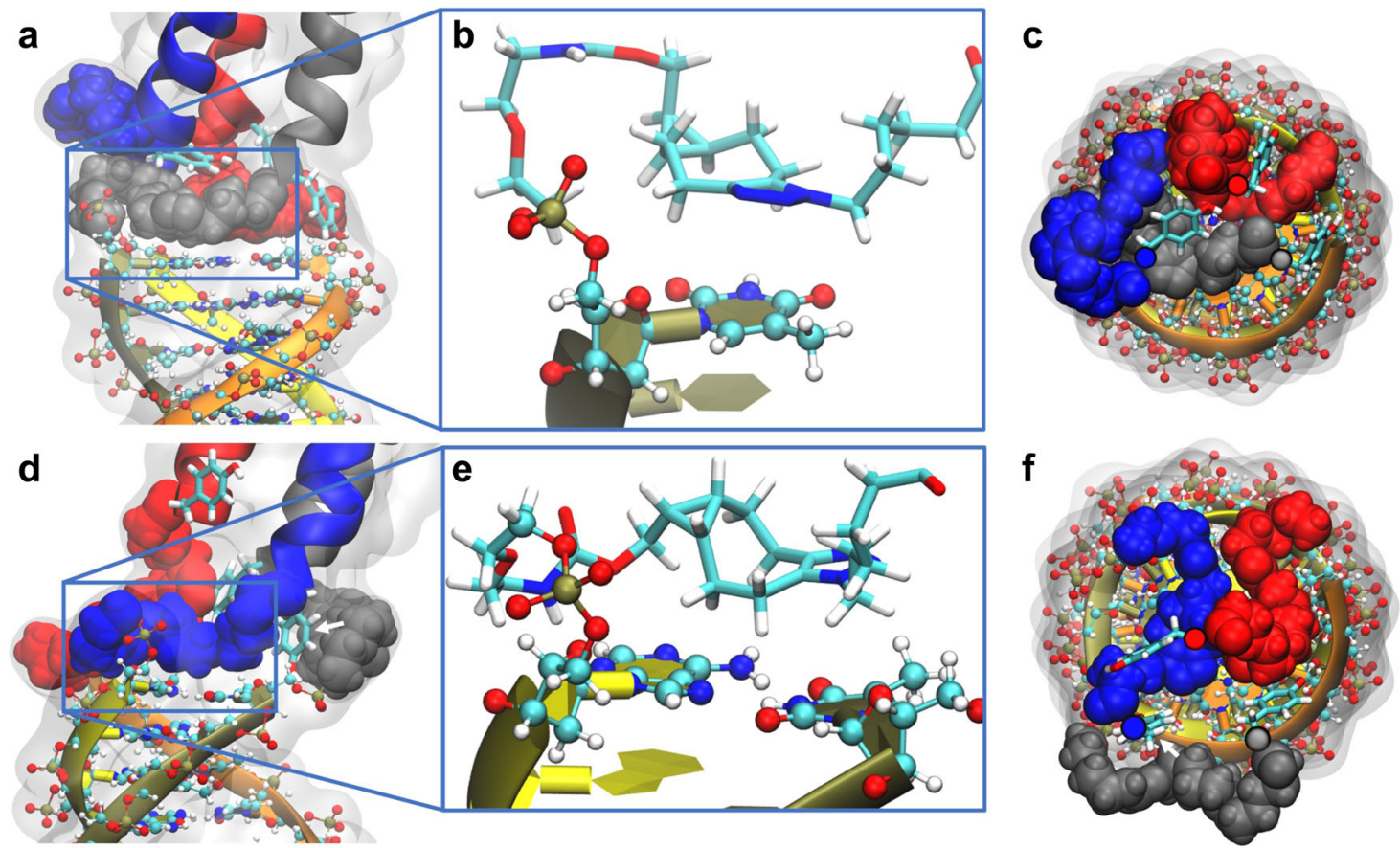

Fig. 5 MD simulation. Packing of the linker region in the MD snapshots used for the SAXS prediction for $\mathbf{D}, \mathbf{D}-\mathbf{P O C}(\mathbf{7}+\mathbf{8}+\mathbf{9})^{\star}(\mathbf{a}-\mathbf{C})$ and for L,D-POC(7 $+\mathbf{8}+\mathbf{9})^{\star}$ of our previous work ${ }^{30}(\mathbf{d}-\mathbf{f})$. a, $\mathbf{d}$ Side views show dynamic stacking interaction between a linker triazole moiety (boxed grey in (a), boxed blue in (d)) and DNA triplex terminal bases. Terminal tyrosines are shown in stick representation. DNA atoms are shown in ball and stick representation. $\mathbf{b}$, e Isolated views of the linker/base stacking interactions where the linker is shown in stick representation. c, $\mathbf{f}$ Top views along coiled-coil axis showing packing of the linkers and tyrosine side chains. Except for the tyrosines, coiled-coil atoms are not shown but collectively represented by the $\mathrm{C}$-alpha atoms of the terminal tyrosines (red, blue, grey spheres with black outlines). White arrows indicated the tyrosine side chain which transiently separated one linker strand (grey) from the other two hydrophobically packed linker strands (blue and red) in the chosen snapshot.

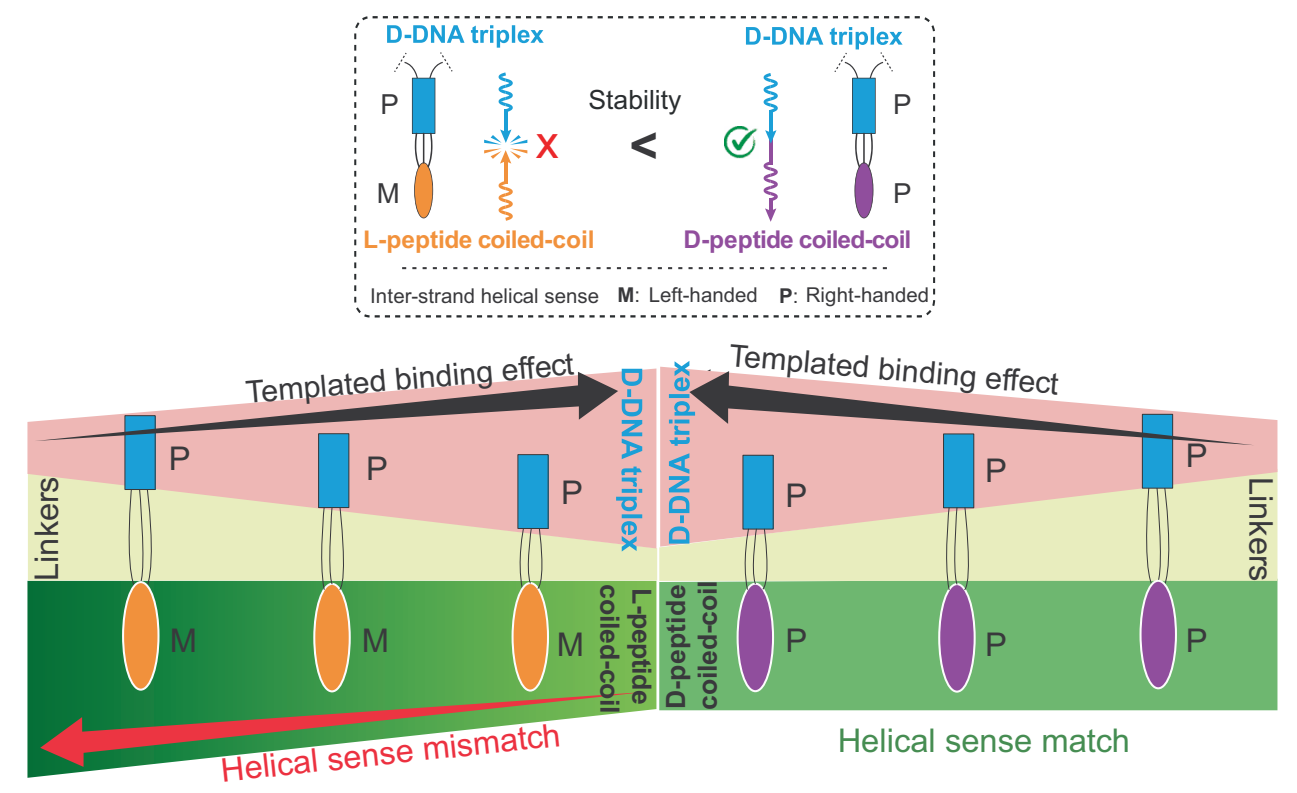

Fig. 6 The stability of POC triple helices is influenced by the helical sense mismatch/match and the templated binding effect. D-DNA triplex is marked in cyan rectangle, L-peptide coiled-coil in orange ellipse and D-peptide coiled-coil in purple ellipse. M and P stand for left-handed and right-handed interstrand helical senses, respectively. (Top: The clash symbol means the helical sense mismatch state, which may result in unfavourable energy cost and thus is marked in a red-cross. The no clash symbol represents the helical sense match state, which should not influence the stability and thus is marked in a green check). 
coiled coils to circumvent the possible chirality clash ${ }^{51}$, which is far beyond the $4.5 \mathrm{~nm}$ limit found here. Another work on a mutated version of Yersinia YadA stalk domain (S299-L362) also showed that a 26-mer motif acted as the transition element between the opposite superhelices ${ }^{52}$. The helical sense mismatch effect unveiled in the present work lends support to the emergence of screw-sense homopairing (or homohelicity) between neighbouring macromolecular domains.

\section{Methods}

Synthesis of DNA constructs for single-molecule investigations. A polythymine (T90) containing oligonucleotide was taken and annealed with two POCs at the $5^{\prime}$ - and $3^{\prime}$-end (Fig. 1). The annealed construct was phosphorylated to insert phosphate groups at the $5^{\prime}$-end of a POC. Then, it was ligated with two nonpalindromic (1558 bp and $2424 \mathrm{bp}$ in length) double-stranded DNA handles. The $1558 \mathrm{bp}$ DNA handle was synthesized from PCR strategy by using a pBR322 plasmid as template, and 5'-biotin-TEG-GCA TTA GGA AGC CCA GTA GG and 5'-AAA CCA TAG AGG CTA CAC TAG AAG GAC AGT ATT TG-3' as primers. BsaI-HFv2 enzyme was used to digest the purified PCR product to generate a sticky end compatible with the annealed fragment prepared above. For the $2424 \mathrm{bp}$ dsDNA handles, we first prepared 2391 bp double-stranded DNA handles by PCR using the $\lambda$-DNA template with two primers, $5^{\prime}$-AAA AAA AAG AGC TCC TGA CGC TGG CAT TCG CAT CAA AG and 5'-AAA AAA AAG GTC TCG CCT GGT TGC GAG GCT TTG TGC TTC TC. Then, the purified PCR product was digested by SacI followed by labelling of digoxigenin-dUTP at the $3^{\prime}$-end SacI overhang by using terminal transferase enzyme for $8 \mathrm{~h}$ at $37^{\circ} \mathrm{C}$. To generate a sticky end, a BsaI enzyme was used to digest the purified digoxigenin labelled product. The purified BsaI digested handle and a $33 \mathrm{bp}$ dsDNA adaptor were ligated by T4 DNA ligase to obtain the 2424 bp dsDNA handle with a sticky end that is compatible with the polyT containing fragment. Finally, three-piece ligation reactions were carried out between the polyT containing fragment and two nonpalindromic handles by using T4 DNA ligase, and the final ligated DNA construct was stored in the $-20^{\circ} \mathrm{C}$.

Single-molecule force ramping experiments. A dual-beam laser-tweezers instrument was used to perform the single-molecule experiments ${ }^{53}$. All the experiments were performed at $23^{\circ} \mathrm{C}$ in $10 \mathrm{mM}$ MES buffer containing $100 \mathrm{mM}$ $\mathrm{KCl}$ (pH 5.5). First, the DNA construct was incubated with digoxigenin-antibodycoated polystyrene beads (diameter $2.1 \mu \mathrm{m}$ ). Then, DNA constructs immobilized beads and streptavidin-coated (diameter $1.87 \mu \mathrm{m}$ ) beads were separately captured by two laser traps. Affinity linkages of biotin/streptavidin and digoxigenin-antibody/digoxigenin were used to tether the DNA construct between two beads by bringing two beads in contact with each other. One of the laser foci was fixed while the other was moveable by controlling the direction of the laser beam. When the two beads were moved apart, the DNA tether was stretched to generate tension on the coiled-coil peptide complex. The tension produced on the coiled-coil peptide complex was recorded in the force-extension (F-X) curve through LabView program (National Instruments, Austin, TX) at $1 \mathrm{KHz}$ with loading rate of $5.5 \mathrm{pN} / \mathrm{s}$ (in the $10-30 \mathrm{pN}$ range). Whether the tethered molecule was single or not was confirmed by the $\sim 65 \mathrm{pN}$ plateau in the F-X curve due to the melting of duplex DNA handles. The Savitzky-Golay function was used to filter the collected data at a time constant of $10 \mathrm{~ms}$ in the Matlab program (The MathWorks, Nattick, MA). The unfolding forces revealed in the F-X curves were used to determine the mechanical stability and the percentage of formation of coiled-coil peptide complexes.

Change-in-contour-length $(\boldsymbol{\Delta} \boldsymbol{L})$. During the unfolding of coiled-coil peptide, the expected $\Delta L$ was calculated by the general Eq. (1),

$$
\Delta L=L-x
$$

where $L$ is the contour length of the $(\mathrm{T})_{90}$ fragment which can be determined as, $L=L_{\mathrm{nt}} \times 90 \mathrm{nt}=\sim 40.5 \mathrm{~nm}$, here $L_{\mathrm{nt}}$ is the contour length of a single nucleotide, $0.40-0.45 \mathrm{~nm} / \mathrm{nt}^{54-56}$, and $x$ is the end-to-end distance, which is the sum of the diameter of dsDNA $\left(\sim 2 \mathrm{~nm}^{57}\right)$ and length of the flanking $(\mathrm{T})_{2}$ at each side of the $(\mathrm{T})_{90}$ fragment $(0.9 \times 2 \mathrm{~nm}=1.8 \mathrm{~nm})$, i.e., $2 \mathrm{~nm}+1.8 \mathrm{~nm}=3.8 \mathrm{~nm}$.

Therefore, the expected change-in-contour-length for a coiled-coil peptide complex is $\sim 40.5-3.8=\sim 36.7 \mathrm{~nm}$.

Data analysis. At a force $(F)$, the change in extension $(\Delta x)$ was calculated by the extension difference between the stretching and the relaxing traces at that force. Then, by using wormlike-chain (WLC) model (Eq. (2) $)^{58,59}$, the resulting $\Delta x$ value at this force was converted to the change-in-contour-length $(\Delta L)$ as follows,

$$
\Delta x / \Delta L=1-1 / 2\left(k_{\mathrm{B}} T / F P\right)^{1 / 2}+(F / S)
$$

where $k_{\mathrm{B}}$ is the Boltzmann constant, $T$ is absolute temperature, $P$ is the persistent length of dsDNA $(50.8 \mathrm{~nm})$, and $S$ is the stretching modulus $(1243 \mathrm{pN})^{60}$.
Calculation for relative contributions of chirality clash effect and templated binding effect. We assumed that the chirality clash effect led to the D-peptide/D-DNA system being favoured with respect to the L-peptide/D-DNA system. In the maximum templated effect for the L-peptide/D-DNA (defined as 100\%), the percentage of triplex formation should be equivalent to that of the D-peptide/D-DNA system at each linker length. If there was a $100 \%$ chirality clash effect in the L-peptide/D-DNA, then its triplex population would be reduced to zero. By comparing the percentage formations of the two diastereoisomeric POC triplexes, the normalized chirality clash contribution for the L-peptide/D-DNA system at each linker length was determined by using Eq. (3).

$$
\text { Normalized chirality clash contribution }=(x-y) / x
$$

where $x$ and $y$ are the triplex formation percentages of the D-peptide/D-DNA and Lpeptide/D-DNA systems, respectively, at a particular length of the interdomain linker.

Then, the normalized templated binding contribution was determined as follows (Eq. (4)),

Normalized templated binding contribution $=1-$ Normalized chirality clash contribution

Statistical data analysis. Data shown in Fig. 4B was obtained from different individual molecules. The data were presented as mean \pm SD for 4 sets of independent samples. The statistical data analysis was performed by using two-sided unpaired $t$-tests.

Reporting summary. Further information on research design is available in the Nature Research Reporting Summary linked to this article.

\section{Data availability}

All data are available in the main text or the Supplementary Materials.

Received: 6 November 2020; Accepted: 7 December 2021; Published online: 10 January 2022

\section{References}

1. Krautwald, S. \& Carreira, E. M. Stereodivergence in asymmetric catalysis. J. Am. Chem. Soc. 139, 5627-5639 (2017).

2. Neel, A. J., Hilton, M. J., Sigman, M. S. \& Toste, F. D. Exploiting non-covalent pi interactions for catalyst design. Nature 543, 637-646 (2017).

3. Proctor, R. S. J., Colgan, A. C. \& Phipps, R. J. Exploiting attractive noncovalent interactions for the enantioselective catalysis of reactions involving radical intermediates. Nat. Chem. 12, 990-1004 (2020).

4. Jain, V. et al. Chiral cooperativity in helical polymers. Isr. J. Chem. 51, 1067-1074 (2011)

5. Suginome, M., Yamamoto, T. \& Nagata, Y. Poly(quinoxaline-2,3-diyl)s: a fascinating helical macromolecular scaffold for new chiral functions. J. Synth. Org. Chem. Jpn. 73, 87-101 (2015).

6. Yashima, E. et al. Supramolecular helical systems: helical assemblies of small molecules, foldamers, and polymers with chiral amplification and their functions. Chem. Rev. 116, 13752-13990 (2016).

7. Freire, F., Quiñoá, E. \& Riguera, R. Supramolecular assemblies from poly(phenylacetylene)s. Chem. Rev. 116, 1242-1271 (2016).

8. Cobos, K., Quiñoá, E., Riguera, R. \& Freire, F. Chiral-to-chiral communication in polymers: a unique approach to control both helical sense and chirality at the periphery. J. Am. Chem. Soc. 140, 12239-12246 (2018).

9. Cobos, K. et al. From sergeants and soldiers to chiral conflict effects in helical polymers by acting on the conformational composition of the comonomers. Angew. Chem. Int. Ed. 59, 23724-23730 (2020).

10. Ousaka, N., Inai, Y. \& Kuroda, R. Chain-terminus triggered chiral memory in an optically inactive 310-helical peptide. J. Am. Chem. Soc. 130, 12266-12267 (2008).

11. Le Bailly, B. A. F. \& Clayden, J. Dynamic foldamer chemistry. Chem. Commun. 52, 4852-4863 (2016)

12. Lister, F. G. A., Le Bailly, B. A. F., Webb, S. J. \& Clayden, J. Ligand-modulated conformational switching in a fully synthetic membrane-bound receptor. Nat. Chem. 9, 420-425 (2017).

13. Gan, Q. et al. Translation of rod-like template sequences into homochiral assemblies of stacked helical oligomers. Nat. Nanotechnol. 12, 447-452 (2017).

14. Ferrand, Y. \& Huc, I. Designing helical molecular capsules based on folded aromatic amide oligomers. Acc. Chem. Res. 51, 970-977 (2018).

15. Mazzier, D. et al. Parallel homochiral and anti-parallel heterochiral hydrogenbonding interfaces in multi-helical abiotic foldamers. Angew. Chem. Int. Ed. 59, 1606-1610 (2020).

16. Ikai, T. et al. Helix-sense-selective encapsulation of helical poly(lactic acid)s within a helical cavity of syndiotactic poly(methyl methacrylate) with helicity memory. J. Am. Chem. Soc. 142, 21913-21925 (2020). 
17. De Cat, I. et al. Induction of chirality in an achiral monolayer at the liquid/ solid interface by a supramolecular chiral auxiliary. J. Am. Chem. Soc. 134, 3171-3177 (2012).

18. Ślęczkowski, M. L. et al. Competition between chiral solvents and chiral monomers in the helical bias of supramolecular polymers. Nat. Chem. 13, 200-207 (2021).

19. Maurizot, V. et al. Design of an inversion center between two helical segments. J. Am. Chem. Soc. 126, 10049-10052 (2004).

20. Ousaka, N. \& Inai, Y. Induction of a heterochiral helix through the covalent chiral domino effect originating in the "Schellman motif". J. Am. Chem. Soc. 128, 14736-14737 (2006).

21. Tomsett, M. et al. A tendril perversion in a helical oligomer: trapping and characterizing a mobile screw-sense reversal. Chem. Sci. 8, 3007-3018 (2017).

22. Ha, S. C. et al. Crystal structure of a junction between B-DNA and Z-DNA reveals two extruded bases. Nature 437, 1183-1186 (2005).

23. Silberberg, M. \& Amateis, P. Chemistry: The Molecular Nature of Matter and Change, 9th edn. (McGraw-Hill, 2021).

24. Walshaw, J. \& Woolfson, D. N. SOCKET: a program for identifying and analysing coiled-coil motifs within protein structures. J. Mol. Biol. 307, 1427-1450 (2001).

25. Burkhard, P., Stetefeld, J. \& Strelkov, S. V. Coiled coils: a highly versatile protein folding motif. Trends Cell Biol. 11, 82-88 (2001).

26. Lupas, A. N. \& Gruber, M. In Advances in Protein Chemistry. Vol. 70 (eds. Parry, D. A. D. \& Squire, J. M.) 37-38 (Academic Press, 2005).

27. Stoddart, J. F. Mechanically interlocked molecules (MIMs)-molecular shuttles, switches, and machines (Nobel Lecture). Angew. Chem. Int. Ed. 56, 11094-11125 (2017).

28. Xi, Z. et al. Single-molecule observation of helix staggering, sliding, and coiled coil misfolding. Proc. Natl Acad. Sci. USA 109, 5711-5716 (2012).

29. Woodside, M. T. et al. Nanomechanical measurements of the sequencedependent folding landscapes of single nucleic acid hairpins. Proc. Natl Acad. Sci. USA 103, 6190-6195 (2006).

30. Lou, C. et al. Self-assembly of DNA-peptide supermolecules: coiled-coil peptide structures templated by d-DNA and l-DNA triplexes exhibit chiralityindependent but orientation-dependent stabilizing cooperativity. Chem. Eur. J. 26, 5676-5684 (2020).

31. Lou, C. et al. Folding topology of a short coiled-coil peptide structure templated by an oligonucleotide triplex. Chem. Eur. J. 23, 9297-9305 (2017).

32. Lou, C. et al. Peptide-oligonucleotide conjugates as nanoscale building blocks for assembly of an artificial three-helix protein mimic. Nat. Commun. 7, 12294 (2016).

33. Ogihara, N. L., Weiss, M. S., Eisenberg, D. \& Degrado, W. F. The crystal structure of the designed trimeric coiled coil coil-VaLd: implications for engineering crystals and supramolecular assemblies. Protein Sci. 6, 80-88 (1997).

34. Pandey, S. et al. Chaperone-assisted host-guest interactions revealed by singlemolecule force spectroscopy. J. Am. Chem. Soc. 141, 18385-18389 (2019).

35. Leitner, D., Schröder, W. \& Weisz, K. Influence of sequence-dependent cytosine protonation and methylation on dna triplex stability. Biochemistry 39, 5886-5892 (2000).

36. Petrov, A. S., Lamm, G. \& Pack, G. R. The triplex-hairpin transition in cytosine-rich DNA. Biophys. J. 87, 3954-3973 (2004).

37. Frank-Kamenetskii, M. D. \& Mirkin, S. M. Triplex DNA structures. Annu. Rev. Biochem. 64, 65-95 (1995).

38. Mergny, J. L. et al. Sequence specificity in triple helix formation: experimental and theoretical studies of the effect of mismatches on triplex stability. Biochemistry 30, 9791-9798 (1991).

39. Keith, R. F. Targeting DNA with triplexes. Curr. Med. Chem. 7, 17-37 (2000).

40. Nyman, M. \& McQuade, L. In Trends in Polyoxometalates Research. (eds. Ruhlmann, L. \& Schaming, D.) 151-170 (Nova Science Publishers, Inc., 2015).

41. Selvam, S., Koirala, D., Yu, Z. \& Mao, H. Quantification of topological coupling between DNA superhelicity and G-quadruplex formation. J. Am. Chem. Soc. 136, 13967-13970 (2014).

42. Shoulders, M. D. \& Raines, R. T. Collagen structure and stability. Annu. Rev. Biochem. 78, 929-958 (2009).

43. Bella, J. Collagen structure: new tricks from a very old dog. Biochem. J. 473, 1001-1025 (2016).

44. Weis, W. I. \& Drickamer, K. Trimeric structure of a C-type mannose-binding protein. Structure 2, 1227-1240 (1994).

45. Solmaz, SozanneR., Chauhan, R., Blobel, G. \& Melčák, I. Molecular architecture of the transport channel of the nuclear pore complex. Cell 147 590-602 (2011).

46. Xu, Q. \& Minor, D. L. Jr. Crystal structure of a trimeric form of the KV7.1 (KCNQ1) A-domain tail coiled-coil reveals structural plasticity and context dependent changes in a putative coiled-coil trimerization motif. Protein Sci. 18, 2100-2114 (2009).

47. Vestergaard, B. et al. Bacterial polypeptide release factor RF2 is structurally distinct from eukaryotic eRF1. Mol. Cell 8, 1375-1382 (2001).
48. O'Shea, E., Rutkowski, R. \& Kim, P. Evidence that the leucine zipper is a coiled coil. Science 243, 538-542 (1989).

49. Harrison, S. C. A structural taxonomy of DNA-binding domains. Nature 353, 715-719 (1991).

50. Hartmann, M. D. et al. $\alpha / \beta$ coiled coils. eLife 5, e11861 (2016).

51. Leo, J. C. et al. The structure of E. coli IgG-binding protein D suggests a general model for bending and binding in trimeric autotransporter adhesins. Structure 19, 1021-1030 (2011).

52. Alvarez, B. H. et al. A transition from strong right-handed to canonical left-handed supercoiling in a conserved coiled-coil segment of trimeric autotransporter adhesins. J. Struct. Biol. 170, 236-245 (2010).

53. Mao, H. \& Luchette, P. An integrated laser-tweezers instrument for microanalysis of individual protein aggregates. Sens. Actuators B Chem. 129, 764-771 (2008).

54. $\mathrm{Yu}, \mathrm{Z}$. et al. ILPR G-quadruplexes formed in seconds demonstrate high mechanical stabilities. J. Am. Chem. Soc. 131, 1876-1882 (2009).

55. Mills, J. B., Vacano, E. \& Hagerman, P. J. Flexibility of single-stranded DNA: use of gapped duplex helices to determine the persistence lengths of poly(dT) and poly(dA). J. Mol. Biol. 285, 245-257 (1999).

56. Tinland, B., Pluen, A., Sturm, J. \& Weill, G. Persistence length of singlestranded DNA. Macromolecules 30, 5763-5765 (1997).

57. Rich, A., Nordheim, A. \& Wang, A. H.-J. The chemistry and biology of lefthanded z-DNA. Annu. Rev. Biochem. 53, 791-846 (1984).

58. Baumann, C. G., Smith, S. B., Bloomfield, V. A. \& Bustamante, C. Ionic effects on the elasticity of single DNA molecules. Proc. Natl Acad. Sci. USA 94, 6185-6190 (1997).

59. Yu, Z. \& Mao, H. Non-B DNA structures show diverse conformations and complex transition kinetics comparable to RNA or proteins-a perspective from mechanical unfolding and refolding experiments. Chem. Rec. 13, 102-116 (2013).

60. Dhakal, S. et al. Structural and mechanical properties of individual human telomeric G-quadruplexes in molecularly crowded solutions. Nucleic Acids Res. 41, 3915-3923 (2013).

\section{Acknowledgements}

The VILLUM FONDEN is thanked for funding the Biomolecular Nanoscale Engineering Center (BioNEC), a VILLUM center of excellence, grant number VKR18333 (C.L., K.J.J. and J.W). H.M. thanks the National Institutes of Health [NIH 1R01CA236350] for biophysical evaluations; and National Science Foundation [CBET-1904921] for DNA construct preparations and instrument maintenance and usage. Joan Hansen and Tina Grubbe Hansen are thanked for technical assistance on oligonucleotide synthesis and purification. Kasper K. Sørensen is thanked for technical assistance on peptide synthesis and purification. Josephine Tuborg Boesen and Peter W. Thulstrup are thanked for the assistance on the CD measurement.

\section{Author contributions}

C.L. and H.M. conceptualized the research and designed the experiments; S.P., S.M. and C.H. conducted the single-molecule experiments; M.B.D. carried out synthesis of the nucleoside monomers and special solid support; K.J.J. and J.W. provided the peptides and ONs; C.L. and S.S. performed POC synthesis and CD measurement; S.S. did the nondenaturing gel electrophoresis, A.B. and T.B. conducted ESI-MS characterization of ONs ON-BCNs and POCs; N.J.C. carried out the MD simulation and SAXS fitting, A.V.K. performed the SAXS measurement and raw data analysis, S.P., S.M., J.W., C.L. and H.M. carried out the result analysis; C.L. and H.M. supervised the execution of experiments; S.P., S.M., M.B.D., C.L. and H.M. wrote the original draft. All authors contributed to finalizing the manuscript.

\section{Competing interests}

The authors declare no competing interests.

\section{Additional information}

Supplementary information The online version contains supplementary material available at https://doi.org/10.1038/s41467-021-27708-4.

Correspondence and requests for materials should be addressed to Chenguang Lou or Hanbin Mao.

Peer review information Nature Communications thanks Carlos Bustamante, Haydyn Mertens and the other anonymous reviewers for their contribution to the peer review of this work.

Reprints and permission information is available at http://www.nature.com/reprints

Publisher's note Springer Nature remains neutral with regard to jurisdictional claims in published maps and institutional affiliations. 
(c) (i) Open Access This article is licensed under a Creative Commons Attribution 4.0 International License, which permits use, sharing, adaptation, distribution and reproduction in any medium or format, as long as you give appropriate credit to the original author(s) and the source, provide a link to the Creative Commons license, and indicate if changes were made. The images or other third party material in this article are included in the article's Creative Commons license, unless indicated otherwise in a credit line to the material. If material is not included in the article's Creative Commons license and your intended use is not permitted by statutory regulation or exceeds the permitted use, you will need to obtain permission directly from the copyright holder. To view a copy of this license, visit http://creativecommons.org/ licenses/by/4.0/.

(C) The Author(s) 2022 